Revista de Filosefía, N 26-27, 1997 - 2-3, pp. 39-60

\title{
Materia y generación en Tomás de Aquino
}

\section{Matter and Generation in Thomas Aquinas}

\author{
Antonio Pérez Estévez \\ Univergidad del Zulla \\ Maracaibo - Venezuela
}

\section{Kesumen}

Este artfeulo intenta aclarar el significado de la expresión utilizada por Tomás de Aquino: "las fombas naturales son educidas a partir de la potencia de la materia". Con tal fir, el autor explica, en primer lugar, que la materia primta es pura potencia, es decir, un no-ente que está en potencia para recibir formas; las formas, en consecuencia, le vienen a la materia no de sla potencia sino de fuera de la materia. En segundo lugar, las formas naturales en la generación son producidas de diferentes maneras, pero sicmpre lano la causa engendrante como las formas engendradas provienen de fuera de la matcria. La causa eficiente o engendrante, a su vez, produce las formas con la aylida de un agente exremo o cuerpo celeste: Dios sólo crea el alma humana. "Las fornas naturales son educidas a partir de la potencia de la materia" significa sólo que todo cambio sustancial y generación de un ser vivo se produce a partir de una causa malcrial; si no fucse así, cse cambio sustancial y gencración sería más bien una creación, es decir, sería la producción de una sustarcia a partir de lo nada.

Palabras clave: Tomós de Aquino, materia, generación.

Recibids: 01-07-97 - Aceptado: 15-09-97

1 Este trabajo fonna parte de una investigación que hă sido subvencioridita por el CSIC. 


\section{Abstract}

The intention of this article is to clarify the meaning of the expression used by Thomas Aquinas, "natural forms are educed from the potency of mattcr". With this in mind, the author first explains how primary matter is purc potency, that is, a nonbeing that is in potency to veceive form; forms thercfore come to matter not through its own potency but from outside matcr. Secondly, natural forms in their generation arc produced in different ways, but in cach case both the gencrating causc and the form generated come from outside matter. The efficicnt cause, or generating cause, at the same timc, produces the forms with the help of an extcrnal agent or celestial hody; God only creales the tuman soul. "Natural forms are educed from the potency of matter" means only that all change of substance and generation of living beings are produced from a material cause; if this was not so, this change of substance and generation would in fact be creation, it would be the production of something out of nothing.

Key wards: Thomas Aquinas, matter, generation.

\section{Introducción}

"Pare Averroce, entonces, toda créación produccion o generución es, ciertamente, una educción de las formas que estan en la materia. Al querer rechazar el platonismo de sus antecesores ro confa mis remedio que poner las formas en ta materia misma, ya que no existen separados. E5tando en elto comto simple potencialidial, sic educción, esto es, su extracción, significaha st actualización... La materia e's condición necesaria pera toda produccion. para soda creacion. Jero no es elia ta gue edtace. la que hace salir de sí misma las formas que está contcridas cn cila polencialmenic, ponque su potencia ex puramente pesivive. Requiere ta accion del agenter, al gate Averroes, en diversos pasajes del Talulfut, identifica con Dios". (El destacado del lexto es ruestro).

Este texto está tomado de Rafael Ramón Guerrem, "Sobre cl Conecpto de Makria tr Avcrecs". Al Encuentro de Averroes, Editorial Trotca, Madrid, 1993, p. 89. Por este texto vemos que Ramón Guetrero afima que Averoes sostiene que toda crcáción, producción o generación es una cducción de las formas que están contenidas en la materia; una muleria que, por tanto, contiene en su polencia numerosas formas, a las que un agente externo debe sacar de ia potencia de la materia, ya que esta potencia es puramente pasiva. No es mi intención hacet análisis o crítica alguna de 
este texto. pues no soy expero en Avermes. Lo cito simplemente para poder comprender con precisión los textos que más tarde va a citar a propósito de lonas de Aquino.

En efecto, en la página siguiente, p. 90, va a alimar que "un dostacado iniembro de Ia 'derceha aristutélica', Tomas de Aquino, también sostiene que la producción es una educción de las fomas que cstán en potençia en la materia, como podemos leer en los textos siguientes". Para probar esta opinion ofrece dos textos: uno de la Summa Contra Gentiles (II,86) y otro de Qrusestio Disprtata de Potentia q. IlI, a. 8, c. Y contisua: "Para Tomás, el acto se cxtrac de la potencialidad de la materia, cxcepto en lo que se refiete al alna liumana, que es la t́nica forma creada por Dies. Pura cl de Aquino, todo acto existe previamente en polencía en la matcria; de ahi que su producción sea una educción: 'omnis actus materiae videtur educi de potentia matcriae" (Strmma Theologica, I,9.90, a. 2 y 3). Busqué cstc coxto de la Summa Theologica (I,q.90,a.2) y descubrí que se trara del 20 . "Vjuetur quod non' en donde justajnente aparece como difeculad la doctrina que ét no sustenta sino la de algún otro autor, a la que intcntará dar solución en las respucslas que vicner despucs del cuerpo del atciculo o respondeo ${ }^{2}$. En efecto, en ad secundum trata de resolver la dificultad antcdicha de que "todo acto de alguna materia parece educirsc o extracrsc de la potencia de la materia" diciendo que "extraer un acto de la potencia de la mareria no es otra cosa que algo, que antes estaba en potencia, se convierte en acto ${ }^{3}$. Educir o extract de la potencia de la matcria no signifjea otfa cosa que pasar a acto algo que antes csta en potencia cn alguna matcria 0 , como arima cn otro texto, 'producir por transinutación de alguna materia' 4.

Rafael Ramón Guerrero parece centender la expresión ceducir formas de la po. tencia de la matcria' como extraer las fomas naturales, ituenos la forma humana, de la potencia cncerrada en la materia como si la potencia de la materia fuera como una sucrte de contincnte a depósito de formas en potencia de dunde van a ser extrádas para ser actualizadas por un agentc cxterro. Pienso que tal lectura de Tonís de Aquino no responde al pensarniento tomista de una separacion o distinción real cnte materia/potencia y actolfortu. La potencia pasiva de la materia tomista yue es su

2 Summa Theologica, I, 9.90 ,a.2,No.2:pruetcrea,omnis actus materiac alicuius videtur educi de potcrtid misteriae; curı enim materia sit potentía ad aclum, uctus quilibet precexiscit in materia in potentia.

3 Summa Thesloyica, I, 9.90, a.2, ad 2um:dicendum quad actum extrahí de potentia materine rihil aJivd cest quatm aliquid fjeri actu, quod privs crat in potentia.

4 Summa Theologica, I,9,90,a.3,c.:...quia animil mulonatis non potest produci per transmutarionem a] licuius malcriae, ideo non potes! produci risi a Deo immediate. 
esencia dpuede encerrar en sí misma formas en potencia que puedan actualizarse? Según el Aquinate, ¿pucde la potencia o esencia de la materia convertirsc en forma? iNo queda, cn cse caso, borrada la esencial diferencia entre materia y foma, potencia y acto?

¿Se trata, en la exprcsión 'extraer de la potencia de la materia, educi ex poientia materiae', ciertamente de la 'potencia pasiva' do la 'materia prima'? Ni potencia ni materia son téntinos univocos, con un solo significado, en Tornás dc Aquino. ¿De qué potencía y de qué materia se trata en los textos aludidos que se reficren a la generación de formas vegetativas y sensitivas? ${ }_{i}$ Se trata, como parece sostener Ramón Gucruero, de potencia pasiva y de materia prima?

La lectura que Rafael Ramón Guerrero parece hacer de los textos tomistas nos ha producido primero sorptesa y confusión; despues, nos ha llevado a escribir este trabajo sobre 'matería y gencración cn Tomás de Aquino'. Esperamos quc, al rérntino de este articulo, podamos entender mejor la expresión 'educir o exuraer formas de la potcrucia de la materia'.

\section{La materia en Tomás de Aquíno}

'Materia', ontológicamentc hablando, ticne en Tomás de Aquino, al menos, dos sentidos: cl primero significando 'materia prima', makeria primera y que quiere responder al concepto arjstotélico de prote hyle, una y única pars todo cambio sustancial; el segundo tomo 'materia propia' o apropiada, significando un compuesto sustancial, distinto en cada caso, que se encuentra en potencia o disposición inmediata para recibir esta nueva forma sustancjal o accidental'. A csta segunda matcria solían llamar los medicvales 'matcria sceunda', 'matcria segunda'.

\section{I1.1. Maleria prima}

La materia es, con la forma, uno de los dos clementos constituyentes de las sustancias naturales que son las únicas realidades subsistentes que existen en el mundo de la naturaleza que nos rodea. La materia es el elentcnto potencial, es dccir, el elemento que no es acto sino que liene la disposición de recibir el acto de la forma con el que se constituye la sustancia. El ser sustancial proviene de la forma, que es

5 In Duodecim Libros Metaphysicorum Arigtotelis Expositio, No. 1730:ex his igitur, quac hic dicuntur, accipitur guod prima materia est una onnium generabilium et corruptibilium; sed propiac materiac sunt diversae diversarum. 
acto; de la materia provienc la potencia o disposición de recibir formas o actos formales, $o$, dicho de otra manera, de la materia provicne la disposición de cambiar.

Matcria prima oquivale a matcria informe o matcria sin forma alguna. Tal materia es, de suyo, tokalmente opaca e incognoscible por absolutamente indeteminada y potencial, debido a que la forma es el principio de todo conocimicnto y la que, con su acto, determina a la materia y la informa para, juntas, constimir una sustancia cspecifica ${ }^{\circ}$. La materia de suyo incognoscible, la conocemos, según Tomas de Aquino, indirectamente de dos nameras distintas: por.analogía y por su relación con la forma?

Conocemos la materia por analogía o semejanza cuardo, en los cambios sustanciales, a Iravés del conocimiento de las formas que cambian llegannos a] conocimiento de algo que perdura y a lo que llamamos matcria. De manera semejante a como percibimos quc un trozo de madera se convierte et una cama o lecho, sicut ligntum ad lestum, percibimos que un tronco se convierte en ceniza, quc un cucroo muerto se convierte en polvo, que el aire húmcdo, al cnfriatse, se convicrte en agua, que un hucvo se convierte en pollo y que un grano de maíz o semilla se convierte en una planta. Para que csta succsion de forrnas sustanciales o de actos pueda producirse, tiene que haber un sustrato último en el que se dé ese cambio formal o un soporte dc ese cambio sustancial; pues bien, ese soporte o sustrato del cambio sustancial es la materia prima o la materia sin forma alguna ${ }^{8}$. Descubienta la matería prima por medio de exta suerte de razonamiento discursivo o deductivo a partir del proceso continuo de cambios sustanciales, intentanios captarla cn su misteriosa y opaca 're-

6 De Potentia, 9.4, a.1, ad Jum: matcria in sua informitate considerat invisibilis, sccumdum quod carer forma, quac cst omnis cognitionis primipium. No entramos angul tn el coustrutertido problema del conocimicnto que, según Tomás de Aqujno, Dios y los angckes teren de la materia porgue no considcranos que afecte 니 tema de la educción de las formas.

7 In Boetium de Trintate, 9,4,a.2....nateria autern secundum se sit jgnota; non potesl secundun se ex ea accipi divursicas generis, st'd solum illa modo quo cognoscibilis est. Est autern cognoscibilis dupliciter. Lino modo per analogian, sive per comparationtum, ut dicitur in I Physicae; hoc tst, ut dicamus hic esix materiast, wel quod materia hoc modo habel ad res naturalcs, sicur lignum ad lecium. Alirs modo cognoscitur per forman per quam labet esse actu. Unumquodque cnim cognoseitur secundum quod sist actu, non is:cundum quod est in potcntia, ut dicitus 10 Metophysicae.

B In Duodecin Libros Metaphysicoram Aristotelis Expositio, No. I688: cum igitur sit quaedam mutatio sccundum substantian, sciliact generabo el comptio, opurket ess: aliquod commune subjectum, quod subiiciatur contrariis mutationibus secundum generationern ct corruptionem; et boc posicis terminis, qui sunt forma el privatio; ita scilicet quod quardogue sit actu per formarn, et qubndogue sit subjectum privationis illius forme. 
alidad', abstrayéndola de todo aquello que en la sustancia natural provjene de la forma, que es acto formal o ser formal y determinacion especifica. Lo que quede en la sustancia, es la materia prima.

Conovemos también indirectamente la materia en su relación con la forma, per habitudinem ad formam. En efecto, en el conocirniento de toda csencia de un individuo concrcto llegamos al conocimiento de su forma específica, pero una forma específica que dice una esencial relación a su materia.

La materia prima, asi concebida sin forma, no tiene ser alguno, es un no-cnte; pero no es absolutamente la nada, ya que su potencia la dispone para recibir el ser formal ${ }^{9}$. Tomás de Aquino habla de la 'materia prima' unas voces como 'no-ente' y otras ncgando que sca cnte. 'Materia prima, sicut non est ens nisi in potentia', la miaurria prima no es un ente sino en potencia, dice en la $\mathrm{q} .5(\mathrm{a} .3, \mathrm{ad} 3 \mathrm{um})$ de la parte $\mathrm{l}$ de la Summa Theologica. Pero en otros textos afirma que es un no-ente, un no-existenke, pero, a la vcz, advierte que no es absolutamenk nada sino 'que es en potencia $y$ no en acto' (S.Th,1., q.5,a.2ad lum). Usa la expresión de que 'la matería prima es poicncia pura' y llega a afitmar que 'la potencia es la csencia de la matcria prima ${ }^{10}$.

La matcria prima o materia concebida sin forma es una extraña realidad, que siendo un no-ente, un no-existente, sin embargo, no es absolutamente nada sino 'un ente en potencia', "potcncia pura' con capacidad o disposición de recibir formas sustanciales. A exte extraño 'ente en potencia' o 'poterncia pura' lo califica como ente imperfectusimo. Ente imperfectísimo que ni existe en la naturaleza, ni puede existir en modo alguno debido a que Dios no puede crearlo, tal como explícitamente to afirma en el Quodibeto $M$, q.1,a.1,c. Y no puede crearto porque 'repugrta a ta razon de ente un no-ente gue simulianeamente y sejún to mismo exista' y 'Dios no puede hacer que algo sea y no sea al mismo tiempo', es decir, cstas proposiciones son lógicamente imposibles o implican contradicción. En efecto, la materia prima es un-ente; por tanto, no pucde cxistir porque, si existiera, seria al mismo tiempo 'un ente en potencia' y 'un ente en-acto', pucs existis cquivalc al 'acto de ser'. La imposjbílidad de que la materia exista sola, sin la forma cs absoluta y no admite excepćtón alguna o milagro". Por cso, a la objeción franciscana de que 'más depende el

9 Op.Cit., No. 24,37:lertio modo dicitur non eתs ipsa materia, quae, quaniurn est de se, non est ens actu, sed ens polentia.

10 S.Th., b. q.77,a.l,ad 2urn: actus ad quem est in potentia riateria prima, exl tubstantialis forma. Et iden polentia matcriate nos ext aliud quam ejus esichtia.

S.Th,I,q.1 15,a.l ad 2um: sed lioc est treatefia prima, guac est polentia pura...

11 In Duodecim Libros Metaphyytcirum A ristotedix Eyositio, Mariedi, Turin-Rums, 1964, No. 1292: matcria crim non potest per se existere sine forma per quam est ens actu, cum 
accidente de su sujeto que la materia de la forma y el accidente puede existir milagrosamente en la Eucaristia sin su sujcto, lucgo con mayor razón pucde la materia existír sin la forma', Tomás responde que este argumento por analogia no ticne valor alguno porque el accidente es forma y acto -accidentales claro está- y su sujeto desempcña el papel que lo soporta o aguanta; la maicria prima es sólo potencia pura y la forma es su acto y le da el ser actual y sustancial del que participa. Por tarto, no puede darse ente ellos strmejanza alguna ${ }^{12}$. Para Tomós de Aquino, la realidad ontica del accidente es superior a la de la martria por ser acro o forma mientras que la materia notes acto sino pura potencia.

La materia prima no puede ser jamás un 'quod est' o algo que subsiste en cl ser; la forma sin embargo, es aquello por lo que also es, 'id quo aliquid est', pro en las sustancias simples o espirituales es tambien 'quod est', cs decir, en uлa sustancia o subsiste por si misma en el $8 \mathrm{cr}{ }^{13}$.

Hay que recordar en esle momento la doble composición que, según Tomás de Aquino, tienę la sustancias naturales o matcriales: la composición de materia + forma con la que se constituye una sustancia marerial, y la composición de sustancia + acto de ser por la que csa sustancia marerial existe fuera de sus causas. La materia prima o informe es 'potencia pura para recibir la forma sustancial de la que recibe, a su vez, el ser sustancial'; una vez constituida la susiancía natural, o sca, una vez unidas la matcria y la forma, está la sustancia natural en disposición o potcncia para cicibit el acto de ser, por el que esa sustancia natural existe fuera de sus causas. La materia prima o informe está, por tanto, con respecto al acto de ser en una doble potencia; está en potencia, primcro, con respecto a la forma para, juntas, constituir una sustancia natural y, ya formando parte de la sustancia está a su vez en potencia con respecto al acto de ser por el que la sustancia natural existe fuera de sus causas ${ }^{14}$. La

de se sit potentia tanturm. Aquí la razón para que la materia no pueda existir, sc debe a que no puede ser sustancia por no cumplit con los dos tequisitos de loda sustancia, a saber, ser separable y ser indjviduo. No. 1291.

12 Qucell. $/$. q.I. a.l.ciaccidens secundura suum exse dependet a subjecto sicul a causa sustentantc ipsum... Sed materia secundum suum esse actude dependel a.forma in quanIum forma est ipsc aclus eius; unte non est simile.

13 De Spirit. Creat., q. uftica, a.1,ad Burlicet forna possit dici quo atiquid est, tamen mate ris non proprie polest dici quod est. cum non sit misi in potentia. Sed quod cst id quord subsistit in esse, quod... in substantiis incorpore is est ip forma simplex. .

14 De Spririt. Creat, q. unica, 8. l,c:Unde in rebus compositis est considerare duplicem acturn et duplicent potentiarn. Nam pimo quidest frateria cst ut potentia respectu forTnse, et forma est actus cius; et iterum natuja constituta ex materia et forma, est matria et forma, est ut potentia respectu jpsius esse, in quantum est susecptiva cius. 
realidad ontolóbica de la matcria prima tornista, sin ser absolutamente nada, es sumamente imperfecta $y$ encierra una doble polencialidad que la reduce a una cuasi sombra rayana de la nada: no es acto sino sólo potencia como elemento potencial de la sustancia y, ya participardo del ser sustancial, está en potencia con respecto al existir.

Si la maleria no puede existir sola, sin forma alguna, no pudo, al comierzo del mundo, preceder en el tiempo a su formación y a su distinción, pues 'afírmar que la materia precedio a su forma es afirmar un ente-entacto sin acto, lo que es contadictorio ${ }^{15}$. La materia no fue creada por bios temporalmente antes de su formación; fue simplemente concreada con la foma, constituyendo compuestos sustanciales elementales individuales y distintos, tal como se describe en el Génesis ${ }^{16}$. La anteriorioridad de la matería sobre su formación en cl acto de la crcación es una anterioridad lógica o de naturaleza en la medida que el acto deleminante de la forma supone la potencia deterninada de la materia o "aquello de lo que algo se hace es por naturaleza anterior a lo hecho' 17 . Pero, hemos de tener en cuenta que, en términos absolutos, siempre algo en acto o perfecto ha de preceder a algo en potencia o imperfecto, pues el ente en potencia no puecte pasar a acto sino por algún ente en acto li: Dios, acto puro, precedió desde toda la etemidad a la materia o potencia pura la cual fuc concreada con la forma por Dios, constituyendo sustancias naturales, distintes $e$ individualcs.

La materia prima, sin forma, es un no-ente, pero no absolutamente rada; es un 'ente en potencia' o con disposición para recibir formas o actos sustanciales y, a traves de esos actos sustanciales, el acto de ser o existir fuera de sus causas. Como crịe en potencia no-es todarla; como parte de la sustancia natural o material, participa del ser de la sustancia rccibido de la forma. La materia, en el compuesto sustancial, participa del ser sustancial que proviente de la forma. Nunca tiene, por tanto, la ma-

I5 S. Theol. 1,4,66,a, , c:impossibile est dicere quod informilas matcriac tempore praccexserit vel formalionem ipsius vel distinctionem. El de formatione quidem manifestum est. \$i enim materia informis praecessit dutatione, haec erat jam in actu... dicese igitur molerian praccedene sise fotma, est dicerens actu sime acn, quod implicat contradictiontern.

16 Ibidem: unde oportel dicere quod materia prima ncquc fuit creata omnito sine fortha, reque sub forma ura communi, sed sub formis distinctis.

17 De Peventice, q.4,a.l,c: melcria informit... praecessit (fomationern) tartun ordinc qacurac, sccundum quod j]lıd ex quo fil aliqujd, naturalíer est prius $\infty . .$.

18 S.Theol., I, q-4,a.l,ad 2um: Printcipium malcriak, quod apud nos imperfoctum irvenitur. non potest esse simpliciter primum, sod pracesditur ab alio periccto... oportet enim aute id quod est in potertie esse aliquid in actu; cum ens if potentia non reducatur in actum. nisi per aliquod coss in actu. 
tcria ser propio: no participa por sí misma del scr sustancial ni siquicra como clemento constiuycnte de la sustancia natural. El ser de la materia, en el compuesto sustancial, es un ser participado por medio de la forma de donde jo reciba"

I. a materia prima o ínforme es un ente en potencia, un ente con una doble potencialidad, rayana de la nada, pero no es absolutamente rada, pues, al menos, participa de alguna manera de lo bucno aurtque no participe del ente. Participa de lo bueno en la medida que ciende a cl como a su causa final; de aquí que no le conviene a la materia ser apetecible sino apetecer ${ }^{20}$. El bien, transcendental del enle, expresa, sin embargo, una razón distiati de la raz6n de entc, la razon de ser descable yfo desear. La materia, en cuanto no-ente no encicra en sf misma bondad alguna, pero, en cuanto ente cn-potencia, busca y desea el ser bajo Ja razón de bicn deseable.

"La maseria prima, dice texhalmenle el Aquindte, parsicipa del bien, pues tiende hacia $e^{\prime}$ (no se tiende sino stolo a to semejarte); sin embarbo, no participa del ente pues se supone que es fo-ente... El bien se extiende. a las existentes $y$ a los no-existentes, no segun ta predicacion sino segün la causatidoul Por ro-existentes entithdemos rti aquetlas cosas que abso tutchente no-son, sino aquellas conas gate son en potencia y no en acto, porque el bien tiene razón de fin, in et gue nn xibtn descinsun tar cosus gue son en acto sino también hacia el que se mueven tas cosas gue no son sino sólo en potencia.. ${ }^{\text {ant }}$

La materia prima, hemos repetido, es un ente en potcncia, cs potoncia pura. Aclaremos la connotación de esta potencia que acompaña siempre a la materia pri-

19 De Spiritualibus Crealuris, qunica, a.1,c: in natura rerum corporsarum matcria non per so panticipat ipsum esse, sed per formam; forma enim adveriens materiac facł jpsam esse in actu. sicut arima corpori.

De Potentia, q,4,a, l,c; quidquid ertim in renum natura inverihur, actu existih, qubd qujdem non habet matcria risi per forıлatis, quae est actus eius.

20 S. Theol, I, q.5,a.3, ad 3uin: matcria sicul nur ust ens risi in potentia, ita nec bonum nisi iл potertía. Sed tanen participat aliquid de hono, scilicet ipsum ordinem vel aptitudinem ad bonum. Er ideo non conretrit siti quod sil appetibils, sed quod appelat.

21 S. Theot., $, 9.5, a, 2$, ad fur); materia prima participat bonum, cum appelat ipsum (nihil autem appetit nisi simile sibi); non autem participat ens cum ponatur non-cns. S. Theof.r I, 9.5, a.2, tud 2um: boлum extenditur ad exjstentia ct non existentia, non secundum pratedicationem, sed secundur causilitatem: ut per nor existentiz intelligamus, ron es simpliciter quite pernitus non sunt, sed ea quac sust in pownlia et non in actu: quia bonum habe: ralionem finis, in quo non solum quiescunt quae sunt in actu sed ad jpsum eliam moventur quide in accu non sunk, sod in potentia tuntum. 
ma como su esencia. La potencja de la materia se contrapone en pitmer lugar a 'acro', y 'ente-en-potencia' sc contraponc a 'cntc-en-acto'. 'Entc-cn-acto' significa simplemente un 'entc', lo que en la naturalcza significa una sustancia corpóreca. 'Entc-cn potencia' significa un no-ente; pero un no-ente que no-es la nada absoluta site 'algo que. sin ser, está en disposición de recibir el acto de ser proveniente de la forma'. Estc extraño 'ente en potencia', en cuanto no-ente no participa del ente, pero, en su disposición de recibir el acto de ser proveniente de la forma, participa de lo bueno y descable; por eso tícnde y desea, appetit, el ser en su razón de bueno y descable.

A la potencia de la matcria prima se le dice que es una 'potencia pasiva' y se la contrapone a la 'potencia activa' de la forma ${ }^{22}$. La 'potencia activa' o verdadera potencia -de possum, *potere conduce a la acción o a la operación y acompaña siempre a la forma, que cs acto primero; la 'potencia pasiva', esencial a la matcria, conduce al acto primero que es la forma. Nada actúa sino en razon de la forma; nada sufre o recibe una acción formal síno en razón de la materia ${ }^{23}$. A Dios, como acto puro, le convicne co un grado supremo la acción; a la matcria como tpura potencia pasiva' le conviene en grado supremo la pasión o capacidad receptiva de formas, 'ckm enim materia sit in potentia, oponet quad primum principiam materiale sit maxime in potentia; et ita maxime imperfectum' ( $S$. Theol., $\mathrm{I}, \mathrm{g} .4, \mathrm{a} .1, \mathrm{c})$. Dios cs inFinito en su acto puro; la matcría prima cs tambien infiлita en su polcncia pura pasiva a reccptora de fomas, no cjertamente simpliciter sino s6lo secundum quid, pucs puede recibir todas las formas naturales o corpóreas pero no las formas ininateriales o espirituales ${ }^{24}$. La potencia ackiva, caractcrística de toda forma, es capacidad de hacer y de actuar, 'potentia ad agere se tenet ex parte formae quae est principium agendi'; la potencia pasiva, característica de la materiat es capacidad de recibir el acto primero de scr o la forma con la que va a construir un compuesto sustancial; o, como dice también Tomás de Aquino, la potencia de tecibir cl ser, cl ser sustancial, 'potentia ad es'se se tentet ex parte muteriae' (S. Theol., I-U, $\mathrm{Q}-55, \mathrm{a} .2, \mathrm{c}){ }^{25}$.

22 De Potentid, 4.3, a.1, ad 2unt; Ve] potest dicj, quod (mundus) cral possibilc propter po. tentian intivam ingentis, non propter aliquam potentiam passivam materiac (primac).

23 De Potentia, q.l,a.1,c: duplex est potentia: una activa cui tespondet acrus, qui cst operatio; ct hutc primo nomen potentiae videtut fujsse attributuin; alia est potentia passiva, cui respondel actus primus, qui est torma, ad quam similites videtur secusdario nonen potentiac devolutum. Sicut autem nihil patitur nisi ratione potentiec passivae, ita nithil agì nisi ratione actus primi, qui est fotma... D mum; unde ipsi convenit noximc agere...

24 \$. Theol, 1, a.7,a.2,ad 3um: matcria, ctian stcundum potentian, non est in[inita sirspliciter, sed secundum guid: guia cius potentia non sc cxtendit nisi ad fotmas naturales. 
Esta potencia pasiva o casi infinita capacidad seceptiva de formas o seres sustanciales que posee la matcria prima, cs cl origen, cn el mundo natural, de todo cambio, de toda generación y de toda compecion. La fadical imperfección de la matería prima, por su esencial potencia de ser, es la razón material de toda transformación que se produce en la naturaleza. El conjunto de sustancias materiales o corpóreas, que constituye el mundo natural, están compuestas de materia y forma. Por la fortna, ticnden a permanecer en el ser especifico que ticsien, pero por la esencial imperfección de la materia siempre pueden no-ser eso que son para ser otra sustancia distinta; la sustancia material siempre puede no-ser lo que es o perder su forma suslancial, y sicmprc pucde convertirse cn otra sustancja distinta o adquirir una nucva forma sustancial 26 .

Rcsumiendo, diríamos que la 'materia prima' tomista ro significa ninguna realidad cxtramenta] que exista o pueda exiscir. No es un ente existente, pero tampoco es la nada absoluta. Es un concepto elaborado por nuestra mente a partir de los cambios que se producen en los compuestos sustanciales de materia y forma o sustancias naturales y que pretende significar una hipotética rcalidad metafisica quc sin ser la nada absoluta tampoco es un ente; es un no-ente que está en porencia para recibir formas y se encuentra como realidad común oxulta $c$ invisible cn lodo cambio de forma sustancial. Si en esas sustancias vamos abstrayerdo todo aquello que es forma -sustancial y accidental- nos quodarcmos con un sustrato que es 'no-ente o un entc en potencia, con disposición y deseo de recibir formas'. En csta contradiccion de 'un no-ente que es en potentia' se desarrolla toda la concepción tomista de la materia prima.

\subsection{Materia existente y las transmutaciones}

La materia prima ni existe ni puede existir, Ia única materia existente es la matcria informada, como elemento constituyerte de un compuesto sustancial especifíco. Esta materia informada o corporcta cstá dotada de ura caulsidad o dimensiones concretas y posec un tipo de organización de acuerdo a la perfección de la forma que recibe y a las operacioncs que esá forma deberá realizat ${ }^{27}$. A formas más per-

25 S. Theol, 1,q.54,a.3,ad 3um: dicendum quod potentia materiae est ad jpsum esse suhstantiale.

26 De Potentia, q.5. a.l,c: illac solac res in sua natura possibilitatem ad ton essc, in quibus matcria contrarictati subjectn... In illis tebus in quibus cst possibilitas ad non cssc, materia permanct...

27 Quodibetwo $\boldsymbol{X}$. 9-4,a.l,c: Unde oportct omnia quac suni composita ex materia dinum- 
fectas y más simples corresponderán materias informadas o cuerpos cada vez más complejos y complicados, capaces de llevar a caho las operaciones que esas formas necesitarán realizar. El mundo naturaj es un conjunto de sustancias compuestas, ordenadas de acucrdo a las funciones u operaciones que deberán cumplir, en las que la matcria informada o cuerpo scrá tanto más perfecta cuanto lo sca la forsta a la que cstá unida. Las materias informadas de los elementos primeros -agua, tierra, aire y fucgo- son menos perfectos que las materias informadas a cuerpos de una planta, de un perro o de un ser humano, debido a que las fornas comespondientes de los elementos son menos perfectas que la forma vegetativa de una planta, la scnsitiva de un perto o la intelectiva de un ser humano, y las opcracioncs scrán, a su vcz, más clementales y menos perfectas. Desde los elementos primigenios al scr humano, las sustancias makriales van ordenánđose en generos y especies cada vez más perfectos dc acuctdo a la forma correspondiente la que, a su vez, exige una maleria más perfecta o mejor organizada ${ }^{28}$. El cuerpo humano o materia informada es la más perfecta de las tmaterias corporeas tal como corresponde a la forma cspecifica del ser humano o alma intelecriva y a las operaciones tan diversas que, como instrumento, ticne que realizar: desde la operacion vegetativa de comer hasta la espiritual acción de pensat o de querer.

\section{II.3. Transmutaciones o cambios sustanciales}

Todo compuesto sustancial de materia y forma es, por tanto, un compuesto de potencia y acto. El cuerpo con sus dimensiones y accidentes desempena el papel de materia con respecto a la forma que es el prircipio de acción. El compuesto susrancial, por la forma, se manticne en el ser especffico que posce; por la materia y su esencial imperfección, tiende a no-ser lo que es para convertirse er otra sustancia distinta, ticnde a transtnutarse 0 a cambiar sustancialmente. Y esta transmuración no puede producirse por slgura acción intcrna de la forma misma de la sustancia, ya que esa forma tiende a mantenerse en cl scr específico que posee; debe ser un agente extemo por medio de lá acción de su forma quien introduce en esta materia una forma distinta. Este cambio de forma conllevará los carrbios correspondientes en la matcria copórea correspondiente. I a transmutación o cambio suslancial de formas es descrito por Tomás de Aquino como una sucrte de confrontación de energías o potencias activas distintas. La sustancia actual tiende a mantenerse en su ser capecí-

sionata esse...

28 De Spirit. Creaturis, q.unica,d.1,c: talis est utiuscuiusque tei fortentian qualis tequirikr perfeclio eius; mam proprius actus potentiam nequirit 
fico mediante el vigor de su forma actual. Para que se produzca un cambio sustancial, es preciso que una sustancia extcma medjante el vigor de su forma, nayor que el vigor de la forma actual, la desplace y la sustituya, constituyendo una nueva sustancia específicamentc distinta ${ }^{29}$. El agente o causa eficientc de la transmutación, id quod efficit, es una sustancia individual o compuesto extemo, que actúa mediante la forma id quo efficil. A su vez el témino de la transmulación cs una nucva sustancia o compuesto. illud qued proprie fir per se; la forna es aquello por medio dc cuya adquisición se dicc que a]go se hace, illud per cuins adquisitionem aliquid fit ${ }^{301}$. Las transmutaciones sc producer cr la naturaleza de una manera natural nuediante la acción de unas sustancias sobre otras sustancias: ituplica siempre la pérdida de una forma sustancial y la adquisición de una nueva. Toda transmutación supone una thatcria o elemento potencial a partir de la cual el cambio sustancial se produce, ex materiae potentia y csto es precisamentc lo que la distingue de la crcación que es producir a partir de la nada, fieri ex nihilo; cn la creacion, por tanto, sc nicga o sc cliti. na la causa material.

\section{II.4. La generación y la materia}

Por generación se entiende el nacimiento de un individuo sustancial dentro de una especie viviente v.g. el racimiento de un gusano, de un perro, de una oveja e, incluso, de ur hombre. La gereración es una trapsmutición cn la que el agente o causa eliciente produce una sustancia individual sentejante especílicatnente a él: un gusano engendra un gusuno, uл perro engendra un perro, una oveja engendra otra oveja y un hombre engendra otro hombre. Toda generación entraña, igual que toda transmutacion, una matcria a partir de cuya potencia o disposición se va a producir la nueva fonna específica gue va a dar el ser a la iueva sustancia: una causa eficiente o agente distinto de la materia quier va a producir la nueva forma y dar una rueva

29 De Malo, 9.5,a.5, ad 14unl: unus actus in potentia existens, impedit seductionem potentiac in aljum acıutr, undt nisi agers tustit tortius quan virtus formae quae est in malcria, sive quam hubet ex ipsa, sive qualm halset ex conservagti, non feducetur in actunt per agens extcrius; thon enim parkus igtis comumpere potcst magnam aquam... Op. Citadkm.

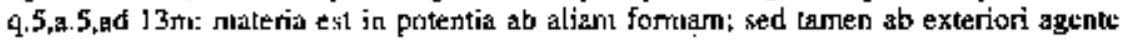
non potest teduci in actum, nisj jllud agens sit fortius quan formas vigor quem habet ex influertia suge formale.

30 De Potentia, g.3,a.8,ce: illud quod propric fet per se, compositum est. Forrma autest ron proprie fit, sed et id qund fit, id esl per cuíus adquisitionem uliquid fil... per naruram ex nihilo nihil fit, quia formas subsiantiales, ex operatinac esse dicamus. Narn id quod ptoprie fit, non est fornus, sed compositum; quod ex malcria tht, el ton ex rihilo. 
disposición a la matcria, y la nucva forma producida o témino de la generacion por la que el nucvo individuo cspecffico se constituye en el ser. Tomás de Aquino distingue dos grandes ajos de generación: la gencración de seres vegetales o plantas y la de animales. En la generación de vegetales o plantas distingue entre la gencración sin semilla a más imperfecta y la generación con semilla o más perfecta. En la generación de animales distingue a su vez tres tipos de generación de acucrdo a la perfección de los animales engendrados: generación de anirnales muy imperfectos, como gusanon, que se engendran a partír de sustancias materiales en putrefaceion, generación de animales más perfectos que se cngendran por medio del coito entre un macho y una hembra, pcro cuya forma o alma sensitiva depende de la materia para cxistir; y. por último, la generación de un indiviouno humano $\mathrm{cn}$ el gue la forma o cl alma subsistentc por sí misma.

Resumamos en un pequeño esquenta:

GENERACION:

1. Generacion de plantas o vegetales:

1.1. Generación de plantas sin poder gonerativo propio, por medio de un cucrpo celeste.

1.2. Generación de plantas con poder generativo asexuado por medio de scmil] i.

2. Generacion de seres sensibles o arimales:

2.1. Generación de animales imperfectos a partir de sustancias en putrefacción.

2.2. Generación de animales más perfectos scxuados.

2.3. Generación del scr hurnano.

No se deticne Tomás de Aquino a explicar con detalle el racimicnto de una nucva planta o vegctal. Hay plantas o vegetales que no poscen fucrza activa capaz de cngendrar; por tanto, son engendradas sin semilla alguna por la cnergía o virtud de otro cuerpo especfficamente distinto, a saber, un cuerpo celeste a partir de una materia converiente o sustancia en putrefacción ${ }^{31}$. I as otras plantas no scxuadas pero con poder generativo propio, poscen unidas tanto la fuerza activa como la pasi-

31 S.Theol, 1,q,92,a.1,c: sumt enim quaedam viventia, quae in seipis not habent virtulem actjvan generationis, sed ab agente alterius speciej generantur; sicut plantae et animalia quas generantur sine semine ex materia convenienti per virtutern activam caclestiusn corएorurn. 
va para engendrar, debido, dice el Aquinate, a que la generación es la acción más noble a la que pueden tender en su vida ${ }^{32}$; ambas fuemas se reúnen en la semilla. La gencración de cstas plantas se produce de la siguiente manera: una planta adulta, o compucsto sustancial dc matcria y forma, cs el engendrante o causa eficientc de la generación de la nucva planta específicarncnte igual ${ }^{33}$, la serrilla encienra en 51 misma canto la matcria a partị de la que la transformación sustancial se va a producir como la virtud activa formal, recibida de la planta adulta, por la que la nueva forma especifica, con la ayuda de la virnd de un cucrpo celestc, se va a producir ${ }^{34}$; la nucva forma especifjea producida da el ser sustancial a la nucva planta o sustancia vegetal. Es de advertir que jamás la materia, por no ser en acto, puede actuar para informarse a si misma; siempre un agente distínto en acto scrá la causa cficiente de la acción que dispondrá a la matcria y engendrará la nueva forma ${ }^{35}$. La forma o alma vegetativa de toda planta depende en su ser y en su hacer de la matcria y se manticne en el ser por la virtud que toda forma posee para mantenerse en su ser, más la ayuda de la virtud de un cuerpo celeste.

En la genemación anirnal Tomás de Aquino distingue, como hemos dicho, tres lipos distintos de generación: en primer ingat, los animales más imperfecros que son engendrados a partir de sustancias en putrefacciór. El extraño caso, para los antiguos, del nacimiento de gusanos en la sustancia putrefacta, lo llevo a echar mano de la virud o poder de un cuerpo celeste para explicar tal gencración. La causa eficiente o cl engendrante, en este caso, es la cnergía o virtud de un cuerpo celeste impresa ton la materia inferior o putrefacta para disponerla a recibir la forma especifica de un animal muy imperfecto. No se cumple en este caso de aninales imperfectos que provience de la putrefacción cl principio de que 'pertencec a la razón del engendrante el que engerdrc algo semcjante a sí mismo según la forma', 'nam de ratione ge-

32 Op. Cis., I,q.92,a.1,6Quacdem vero habent virtutem gencratiunis activam el passivam coniunctam; sicut acoidit in plantis quat gemerantur ex seminc. Nor enim est in plantis aliquod nobilius opus vitae quam gencratio; unde convenienter umni tempots in eis virtuti passivae coniungitur virtus activa generacisnis.

33 S. Theol, $\mathbf{I}, q, 33$, ,, 2 ,ad Aum: de talione generantjs est, quod generet sibi simile secundum Fortram.

34 S. Theol., $11, q .32, a-4, c$ in plantis, in quibus utraque vis (activa ct passivg) comrniscetur, mon est distinctio maris tet feninae.

35 S. Thenl., I,q-32,at.4,c; Nec est possibile quod materia agat ad suj formationem; quia roth est actu. Nec est possibile quod aliquid nowcat sejpsum, nisi dividatur in duas partes, quarum ura sit movens $\mathrm{ct}$ alia mota; quod in snlis animatis contingit... 
neruntis est, quod generet sibi simile secundum fomam' ( $S$. Theol, I, q.33, a.2, ad $4 u m)^{36}$. No existe semejanza especifica o formal entre el cucrpo celeste o causa eficiente de csta generación y el animal imperfecto que nace. Tomás de Aquino va a tratar de explicar csta anomalía diciendo que 'cuanto algo es más imperfecto, tanto menos requisitos se necesitan para su constitución: de aquí que en los animales intperfectos provenientes de la putrefacción es suficiente sólo la virtud o potencia del cuerpo celeste'. Y añade, conociendo lo extraño de tal generacion, que 'si bien no hay semejanza especifica entre el cielo y estos unimales engendrados a partir de in putrefacción, sin embargo existe semejanza en el hecho de que el efecto preexiste viriualmente en su causa activa 3 .

En segundo lugar, los animales más perfectos que engendran sexuadamente por medio del coito entre el macho y la hembra un nuevo individuo de la misma especie. El engendrantc es el macho o el padre ${ }^{38}$ o compuesto de nateria y forma el cual, por medio del semen o virtud activa y con ayuda de un cuerpo celeste, dispone la materia aportada por la nujer: materia que Tomás identifica con la sangre menstrual o sangre especial elevada a más perfecta digestión y preparada por la acción generatriz de la mujes para concebir o recibir la virtud activa o scmen. El semen masculino es como una continuación del padrc y cncierra todo el principio activo y formal miertras que la sangre menstrual femenina desempeña el papel de principio pasivo y material de la generación sexual animal. El semen es descrito como 'la virud o fuerza del alma, la cual aunque inperfecta es una impresión del alma perfectamente dcjada, ${ }^{39}$. La inferioridad del papel de la hembra en la generación es reiteradamente destarcado por 'Ionás de Aquino: 'La potencia generativa de la hembra es imperfecta con respecto a la potencia generativa que posee el varón', dice en un texto ${ }^{40}$. Toda generación es una transmutación de lo imperfecto a lo perfocto, om-

36 Tumbibién en S. Theol., 1,q.4],a.5,c: illud est potentia generativa in aliquo generante, in quo genitum similatur gencrants.

37 De Potentio, q.3,a.l l,ad 12uni; quanto aliquid es imperfectius, tanto ad eiłs constitutionem pauciora रrquinuntur... in animalibss ex putrefactione generatis sufficit sola virtus

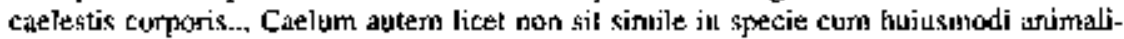
bus ex putrefacrione generatis, est tanen sisnilitudo quartum ad hoc quod effectus is causa activa virtuatiter praeexistit.

38 S. Theol, I, , 41,a.5,c: homo genitus est similis gencranti in natura humana, cuius virtute pater polesl generare hominem.

39 De Potentia, q,3,a,8,ad 14usr: in scnire calor scminis agit ut instrumentum vintatis animac, quac est in seminc; quac quidem licet imperfecta sit, taren est impressio quaedarn animae peffecle relicta.

40 S. Theol., III.q.32,a-4,ad 2um; potentia generaliva in feming est imjerfecta respectu po- 
nis generatio est tranumutatio de imperfecto al perfectom' (De Anima, q.unica, a.2, ad 9um). Por obra de la energía o virtud activa del semen masculino y con la ayuda de un cuepo celesie, la materia, aportada por la mujer y preparada para concebir, se va informando y pasando de potencia a acto en un proceso gradual er e] que cl cuerpo se va perfeccionardo hasta alcanzar la perfección y recibir la foma especifica animal hacia la que la matcria -sangre merstrual- cstaba dispucsta. Así vemos que en la generaciór animal, prímeto se engendra el animal y más tarde el caballo o el hombre ${ }^{41}$. La acción dal varón, como causa cficicnte de la gencración que se produce por medio del semen y de un cuerpo celeste, va perfeccionando la malcria o sange menstrual de la mujer $\mathrm{c}$ introduciendo, gradual y progresivamente, primero la forma vegetariva, más tarde, cuando la materia se ha perfeccionado en un cuerpo, la forma animal, para concluji con la forma cspecífica para la cque c5a matcria estaba dispuesta, a saber, un caballe o un hombre. La úlima forma más perfecta sustituyc a las anteriores menos perfcelas para, una vez constituida la sustancia individual por medio del ser forma], perseverar en el ser ayudada por la influencia de un cucrpo celeste.

La generación del ser humano termina su proceso de transformación por medio de la ercación divina del alma racional. Temina el proceso de gencración del ser humano con la creación por parte de Dios del alma humana; pero el proceso comenzó con el coito del varón y de la mujer. Igual que én los animales perfectos el varón padre aporta el semen o principio acrivo y formal del nuevo ser humano; la mujer aporta la sangte menstrual ,o príncípio pasivo y material a partir del cual el semen, unido a la virtud o energía de un cuerpo oclestc, iniciarś un proceso largo y gradual de información por medio del que la materia se ira perfeccionando ${ }^{42}$, recibiendo. primero, naturalmente por acción de la viturd activa del semen y de una manera imperfecta, la forma vegctativa, Iuego, también de usa manera natural e imperfecta, la forma sersitiva animal y, cuando la materia ha alcanzado la perfección de ur cucrpo humano, recibirá finalmente el alma racional creada por Dios, la cual sustituifá las

tentiat generativas quae est in mate. Er ideo, sicui in artibus ars inferior disponil maleriam, ass autem suphrior inducil forman... ita clian sirtus gencraliva femina praparal materiam, viruss vero activa maris format materiam praeparatan.

41 S.Thent, l,द. 119 , a.2, c: Est autem thaturalis ordo ut aljquid gradatim de potentia reducatur in actum; cr ided in his quae generantur, invesimus quod primo unumquodque est imperfecturi, et postev perficitur... et ideo in gencratione animalis prius gentralur animal, quatm homo cl cquus.

42 Qutudibet $I X, q .5, a .1$, e: dicinus quod anima hominis, quate onmes has potentias anjmas sustinet, a Deo creatur, quarnyis per operationert naturalis agestis fiat ut corpus organizatum accu perficiatur per potentias, çuat sunt corpiralium partiunt actus. 
dos anteriores imperfectas y constituirá el ser del nuevo individuo humano ${ }^{43}$. Las almas vegetativa y sensitiva o animal dependen del cuerpo material en su ser y en su actuar; por eso se producen naturalmerite a partir de la materia que aporta la mujer y por la acción del semen masculino ayudado por la virtud de un cuerpo celeste. El alma humana o racional no depende en su ser del cuctpo naterial pucs posee un set subsistente y continúa cxisticndo sin el cuerpo; cstá nuy por encima de todo orden material, tal como se obscrva en algunas de sus operaciones especificas como la acción totalmente espiritual de pensar. Por cso, neccsita scr creada por Dion y ster introducida en el cuerpo humano.

\section{Conclusión}

Con la nueva pespectiya alcanzada en este trabajo que pretende dar una visión exacta de la posición tomista eл tomo a la materia y en tomo a la generación, regresenos a las expresiones que motivaron cste trabajo cn las que Tomás de Aquino afimaba que 'omnis foma quae educitur in esse per moteriae transmuturionem est foma educta de potentia materiac... ', toda forma gue sale al ser por medio de la transmutación de la materia, es una foma salida de la potencia de la materia (Contra Gentiles, BAC., Madrid, 1967, Liber II, cap.86,p.693), o la que cncontramos en De Potentia (q.3,a.8,c) 'non proprie dicitur quod forma fiat in materia, sed magis quod de materiae potentia educatter, com propiedad no se dice que la forma se hace en la materia, sino mas bien que sale de la potencia de la materia, $\mathrm{e}$. incluso, la $\mathrm{ex}$ presión de la Summa Theologica ( $\mathrm{t}, \mathrm{9} .90$,a.2, videnr2) 'omnis actus materiae alicaius videtur educi de potentio materiae', todo acto de alguna materia parece salir de la potencia de la materia.. Estas expresiones parecen signficar que "las formas Jlegan a scr sacándolas o saliendo de la potencia de la matcria". Para una conceta hemencotica de estos textos, bemes de comenzar leyéndolos en su contexto. Veamos.

E1 primer texto de la Stomma Contra Gentiler (L.U,cap.86) está sacado del capitulo que trata de demostrar que "sl alma humana no sc iransmite por medio del se-

43 Quodlibet $X, \mathrm{q} .5, \mathrm{a} .1, \mathrm{c}$ : animae autem vegetalis est sensibitis thon sunt fornae subsistentes, alias remanerent post corpora: unde oportet quod fiant a generante per actionem conpositorum, sieut ct ceterac formac materiales. Sole autem intellectiva, quae habet esse subsistens, curn maneat post corpus, est ab extrinseco per creationem. Op. Cit. 4.5 , a. 1,ad 2um: quantum pertinet ad hominem, iл cuius sernirne quamvis praecedal vegelativa et sensitiva anima imperfecte, cum illis cessantibus introducatur per creationem anima rationalis, quac perfecte continet qurod in eis erat imperfectionis. 
ment. Formia parte de una demostración en la que leemos: 'toda forma sale al ser por la transmutación de la materia, es una forma salida de la potencia de la materia; en esto consiste la transmutación de la materia, en pasar de potencia al acto. Ahora bien, el alma intelectiva no puede salir de la potencia de la materia, pues como demostró anteriormente el alma intelectiva excede toda la potencialidad de la materia, al realizar alguna operación sin materia, como antes se demostró. Por tanto, el alma intelectiva no sale ul ser por medio de la transmutación de la materia. $Y$, de csa forma, ni por la acción de la virtud que existe en el semen" 44 . Se trata de la generación de un ser humano y, en concreto, de como sale al ser el alma intelectiva. La matería de que se trata en este texto, no es la materia prima o 'un no-ente que es en potencia', sino que es una materia concreta, a saber, la materia que se encuentra en disposición de ser engendrada o concebida por el semen del hombrce-varón won cl fin de hacer nacer un nuevo ser humano. Sabemos que 'csta maleria concreta' es la sangre menstrual de la mujer que provienc del alimento especialmente digerido que la hace apta para la concepción; la materia, en la generación de un ser humano, es una sustancia o compuesto de matería y forma, aportada por la mujer en el acto del coito, la sangre menstrual, dispues!a para recibir el semen del varón e iniciar juntos el proceso generativo del nucvo ser humanto. Pero esta materia concreta o sangte menstrual no encierra en sí forma alguna, a no ser la forma de sangre menstrual, ni liene algún depósito de donde pueda salir o ser sacada la forma humana; esta materia, en cuanto materia, es totalmente pasiva. Simplemente esta maleria se encuentra en disposición de dejar que la virtud o potencia activa de la forma que es el semen, per acrionem virntis quae est in semine, comicncc a actuar sobre ella para transmutarla por medio de un largo proceso hasta llegar a converítla en un ser humano. Fste proceso pasa por un perfeccionamiento gradual de la materia de acuerdo a las nucvas formas que va adquiriendo: como ya hemos dicho, primero se convierte en un ser vegctativo imperfecto, luego en un ser animal imperfecto y finalmente en ser hombre. El alma humana o intelcctiva, que susituye a las anteriores imperfectas producidas por la virtud activa de] semen, no puede provenir de la potencia activa seminal porque está por encima de todo poder material; por $\mathrm{eso}_{+} \mathrm{cs}$ creada direciamente por Dios. La forma no sale ri es sacada a aćto de la potencia pasiva de la materia, sino que la forma

44 Summa Consra Gentiles, lib. II, cap. 86, p. 693: ontnis foma quac cducitur in esst pur

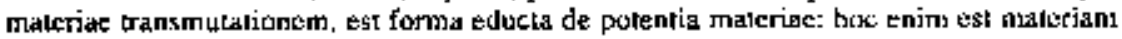
transmulari, th potestiu in actum reduci. Aniuna autem intellectiva non potrst educi de putertia materiae: iam enim supra (7B) ostcnsum csl quod ipsa anina intellectiva excedit lotum pusse matcriae, curn habcat aliquam operationem absque unateria, ut supta bsten-

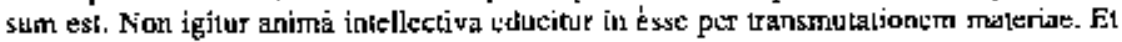
sic, neque per actionem virtulis quar est in semine. 
en toda generación natural, como ya hemos explicado, cstá impresa en el semen el cual, con la ayuda de un cuerpo celeste, da la forma o informa la matería para irla transtrutando y terminar, excepto en el ser humano. por darle la forma definitiva de planta, de caballo o de perro y, a través de la forma, el ser sustancial,

El segundo texto está tomado de la Quaestio disputata, De Potentia ( $\mathrm{q}, 3, \mathrm{a}, 8, \mathrm{c}$ ) de un artículo que versa sobre 'Si Dios actia en la naturaleza al crear; to que significa pregurtarse: si la creación se mezcla con la operación de ta naturaleza $A S$. Forma parte de un párrafo en el que se trata de la producción de una nueva sustancia natural, factio y dice textualmente: 'to producido no es la foma, sino el compuesto: al cual se produce a partir de la materia y no a partir de la nada. $Y$ ciertamente se produce a partir de la muteria, en cuanto la materia está an potencia hacia el compuesio por el hecho de que está en potencia hacia la forma. Y así no decinos con propiedad que la forma se produce en la materia, sino que más bient sale de la potencia de ta materia ${ }^{45}$. Toda producción natural de una nucva sustancia se produce a partir de la maleria y no a partir de la nada. Esa es precisamente la distinctón entre creación y producción natural o transmutación. En la creación se produce una nueva sustancia a partir de la nada, es decir, sin causa material previa, como sucede, por ejemplo, en la creación del alma intelectiva o como sucedio en el origen del universo. En la produccion de una sustancia natural o material se produce el compuesto a partir de la thateria o causa matcrial preexistente $v$.g. a partir de la materia que es el agua se produce una nueva sustancia que es el aire por acción del fuego: $o$ en la generación se produce una nueva planta o un nuevo anínal a partir de una cierta materia concreta que va a ser transmulada por la accion de un agente externo -un cuerpo celeste y e] semer que es la impresión perfecta de la forma de] padre generante- que accúa por medto de su forma. Y producir a partír de la materia significa producir a partir de la potencia pasiva de la nateria, es decir, de la disposición que la materia posee para adquirir tal forma v.g. el agua extá en potencia para adquuiri la forma de aire por la acción del fuego, o la sangre de la hembra está en potencia o disposicion para recibir la forma de animal y de caballo y transmutarse en 'este cabalto blanco'. Por eso, no se dice con propiedad que la forma se produce en la materia o sobre la materia sino que la forma 'sale a partir de la potencia de la materia'; es decir, ś́lo a

45 De Potentiu, 9. 3, a.8, c: utnum Deus operebur in natura creando; quod est quaerere: utnum crestio operi naturae admiscetur.

46 Op. Cit, 9.3, a.8, c: Nam id quod fit, non est forma, sed comporitum; quod cx matcria fit, et mon ex sihilo. Fit fit quidem ex materia, in quantum materis est in polentia ad ip surn compositum, per hor gquod est in potentia ad formam. El sic non propric dicitur quoed forma fiat in malcria, sed magis quod tle malcriac potterlis educatus. 
partir de la potcncia o disposición de csta materia concreta, el agente externo, como e) fuego o el cogendrante, produce la nueva forma y con eIla la nucva sustancia o compuesto.

Veamos por último el texto tercero de la Summa Theologica (1,9.90,a.2, vide(ur 2), que pertencec a un artículo en que se trata de 'si et alma hrmana es producida en el ser por creación', strum anima sil producta in esse per creationem. El videcur quod 2 que expresa una opinión.distinta de la de Tomás de Aquino, es decir trata de demostrar que el alma intelectiva se produce a partir de la potencia de la materia, dice textualmente: 'sodo acto de una materia parece salir de la potencia de la materia; como la materia está en potencia al acto y tado acto preexiste en potencia en la mareria. Ahora bien, el alma es el acto de la mesteria conporat, como se muestra en su definición. For tanto, el alnia sale de ta potencia de la materia, ${ }^{47}$. Anaficemos por tanto la respuesta de Tomás de Aquino a la dificultad expresada en el videtur expuesto: 'extraer (sacar) un acto de la potencia de fa materia no es otra cosa que hacer o producir en acto atgo que antes estabo en potencia Ahora bien, el alma racional no posee un ser dependiente de la materia corporal, pues tiene un ser subsistente y excede la capacidad de la materia comporal tal como se dijo (a. 75,a.2); por tanto, no sale de la potencia de la miateria',48. 'Extrater o sacar un acto de la potencia de la matcria es igual a hacer algo que está en potencia pase a acto' o adquiera una nueva forma por la acción de un agente exterior. En el caso concreto de la generación de un scr humaro la materia concreta es la sangre menstrual de la mujer que esta en potencia para rocibir la nucva forma o almá intelectiva después de un largo proceso generativo, tal como lo explicamos en el cuerpo de este rabajo. Pcro sl alsua intelcctiva posee un ser subsistente propio que no depende de la matcria ni en su ser ni cn su operar, por tanto, no puede provenir de la transmutación dc la materia ni de la acción de ningún agente natural; solo puede ster producida por creación a partir de lá mada.

47 S. Theol., I, q.90, a.2 videtur quod 2 : omnis aclus malcriac alicuius videlur educi de potentia materiae, cum enim malcria sit in potentia ad actum, actus quifibct pracexistit in malesta in potenlią. Sed anima est actus materiac esposalix, ul ex eius detinitione appafet. Ergo anima educitur de poicnlia materiac.

48 Op. Cit, I, 9.90, a.2,ad 2um: actum extrahi de potentia matcriac, nihil aliud esl quam aliquid fieri actu, qund prius erat in potentio. Sed quia anima rationalis non habet esse suun dependens a matcria corporali, sed habłt esse subsistens, el excedit capacitatem maleriake corporalils, ut supra $(4.75$, is.2) diclunt esti propterea non educitur de potentia materiae. 
Resumiendo, podríanos decir que la cxprcsión 'educere fomas de potentia materiae' utilizada por Tomas de Aquino para hablar de transnutaciones o generaciones uaturales, hay que enterderla teniendo en cuenta las siguientes prccisiones:

1. 'Edncere fomas de potentia materiae' ni significa sacar formas o hacer salir formas de la potencia de la materia como dc un lugar, continente o dcpusito en el que las formas están encerradas. La potencia de la materia no cs depósito o un seno matemo de donde las nuevas formas puedan salir a la luz.

2. La materia de la que sc trata no es la matcria prima, imperfectísima o 'un no-entc que es cn potencia' para adquinir todo tipo de formas. La materia, de la que se trata en la transmutación natural y generación, es una materia informada, una sustancia o compuesto de materia y foma con una organjzacion o disposición especifica para adquirit esta nueva forma especifica distinta de la que tiene.

3. 'Educere fomas de potentia materiae' significa simplemente que toda transmutación sustancial natural o gencración se da a pastir de uлa causa material o matcria concreta que se encuentra en potencia inmediata para adquirir una nueva forma concrela específicamente distinta de la que tienc, 'quod (compositum) ex materia fit et non ex nihilo'. Si ba transmutacion sustancial o generación natural se produjese sin una materia previa, estaríamos en presencia de una acción crcativa por la que se produce una forma o un compuesto a parior de la nada:

4. La jueva forma no sale ni se saca de algón pozo o seno, oculto en la poicncia de la materia. La causa eficiente de la nueva forma adqujrida es siempre realmente distinta de la materia. En la generación arimal la nueva forma adquirida es producida, como causa eficiente, por la acción del padre engentrante -a traves de la potercia activa del semen- y con la ayuda de un cuerpo celesic. Hay que scralar la cxccpción de los animales imperfectos que provienen de la putrefacción, cuya causa eficiente es sólo un cuerpo celeste. 